Comment. Math. Helv. 74 (1999) 156-170

0010-2571/99/010156-15 \$1.50+0.20/0
(C) 1999 Birkhäuser Verlag, Basel

Commentarii Mathematici Helvetici

\title{
Platonic surfaces
}

\author{
Robert Brooks*
}

\begin{abstract}
If $S_{O}$ is a Riemann surface with a complete metric of finite area and constant curvature -1 , let $S_{C}$ denote the conformal compactification of $S_{O}$. We show that, under the assumption that the cusps of $S_{O}$ are large, there is a close relationship between the hyperbolic metrics on $S_{O}$ and $S_{C}$. We use this relationship to show that $\liminf _{k \rightarrow \infty} \lambda_{1}\left(P_{k}\right) \geq 5 / 36$, where the Platonic surface $P_{k}$ is the conformal compactification of the modular surface $S_{k}$.
\end{abstract}

Mathematics Subject Classification (1991). 58G99.

Keywords. Eigenvalue, Riemann surface, Ahlfors-Schwarz Lemma.

Let $\Gamma=P S L(2, \mathbb{Z})$ be the group of linear fractional transformations

$$
z \rightarrow \frac{a z+b}{c z+d}
$$

with integer coefficients with determinant 1 , and let $\Gamma(k)$ denote the $k$ th congruence subgroup

$$
\Gamma(k)=\left\{\left(\begin{array}{ll}
a & b \\
c & d
\end{array}\right):\left(\begin{array}{ll}
a & b \\
c & d
\end{array}\right) \equiv \pm\left(\begin{array}{ll}
1 & 0 \\
0 & 1
\end{array}\right)(\bmod k)\right\} .
$$

$\Gamma(k)$ then acts on the upper half plane $H^{2}$ with quotient a hyperbolic surface $S_{k}$ of finite area. According to a theorem of Selberg, we have:

Theorem 0.1. ([Se]) The first eigenvalue $\lambda_{1}\left(S_{k}\right)$ of the Laplacian acting on $S_{k}$ satisfies:

$$
\lambda_{1}\left(S_{k}\right) \geq 3 / 16
$$

In this paper, we will consider a family of compact surfaces $P_{k}$, which we call the Platonic surfaces. They may be described conformally as being obtained

*Partially supported by a Guastella fellowship, the Fund for the Promotion of Research at the Technion, and the M. and M.L. Bank Mathematics Research Fund. 
from $S_{k}$ by "filling in" the punctures of $S_{k}$. For $k=3,4$, and 5 , the surfaces $P_{k}$ correspond to the Riemann sphere with a tesselation by regular spherical $k$-gons. For $k>6$, the surfaces $P_{k}$ carry a similar hyperbolic tesselation, and are thus natural generalizations to hyperbolic geometry of the classical Platonic solids. See $[\mathrm{BFK}]$ and $[\mathrm{SGCC}]$ for some alternate descriptions of these surfaces in terms of graph theory.

In this paper, we will show:

Theorem 0.2. The first eigenvalue of the Laplacian $\lambda_{1}\left(P_{k}\right)$ satisfies:

$$
\lim _{k \rightarrow \infty} \inf \lambda_{1}\left(P_{k}\right) \geq 5 / 36 \text {. }
$$

The number 5/36 arises already in the work of Huxley [Hu] and Sarnak-Xue [SX] in their geometric approach to the Selberg 3/16 Theorem, see also [TFSG]. Indeed, we will prove Theorem 0.2 by showing that the surfaces $P_{k}$ are sufficiently similar to the surfaces $S_{k}$ for the Huxley-Sarnak-Xue argument to apply to them as well.

More generally, we will consider the following situation: Let $S_{O}$ be a Riemann surface with a complete finite-area metric of constant curvature -1 . Then there is a unique compact Riemann surface $S_{C}$ and finitely many points $\left\{p_{1}, \ldots, p_{k}\right\}$, such that $S_{O}$ is conformally equivalent to $S_{C}-\left\{p_{1}, \ldots, p_{k}\right\}$.

A natural question is to relate the hyperbolic geometry of $S_{O}$ with the hyperbolic geometry of $S_{C}$. This would seem at first glance to be problematic, since $S_{C}$ need not in general carry a hyperbolic metric. Even if it does carry such a metric, $S_{O}$ and $S_{C}$ will still have some striking differences - for instance, $S_{O}$ will be non-compact while $S_{C}$ will be compact.

Nonetheless, our main technical result in $\S 2$ below will show that, in the case where all the cusps of $S_{O}$ are large in a sense to be defined in $\S 2$ below, there is a close relationship between the hyperbolic metrics on $S_{O}$ and $S_{C}$ (and, in particular, $S_{C}$ carries such a metric). Namely, there are neighborhoods $\left\{B_{l_{i}}\left(C_{i}\right)\right\}$ of the cusps $C_{i}$ of $S_{O}$ and $\left\{B\left(r_{i}, p_{i}\right)\right\}$ of the points $p_{i}$ which depend only on the size of the cusps, such that outside these neighborhoods the metrics are close.

The main idea in establishing that these metrics are close outside of these neighborhoods is to use a variant of the Ahlfors-Schwarz Lemma [A] due to Wolpert [W], which we will describe in $\S 2$ below.

We will give two applications of this result.

The first one, in $\S 3$ below, shows that, under the assumption of large cusps, the lengths of short geodesics on $S_{C}$ are bounded by the lengths of short geodesics on $S_{O}$. This is the crucial step in applying the Huxley-Sarnak-Xue machinery to the surfaces $P_{k}$.

The second application in $\S 4$ below shows that, under the assumption of large cusps, the Cheeger constants $h\left(S_{O}\right)$ and $h\left(S_{C}\right)$ are bounded in terms of one another

$$
\frac{1}{C(l)} h\left(S_{O}\right) \leq h\left(S_{C}\right) \leq C(l) h\left(S_{O}\right)
$$


by a constant $C(l)$ which tends to 1 as the size of the cusps tends to infinity. It follows from the inequalities of Cheeger $[\mathrm{Ch}]$ and Buser $[\mathrm{Bu}]$ that the first eigenvalues of $S_{O}$ and $S_{C}$ are bounded in terms of one another.

In [BBD], a different method was employed to compactify the surfaces $S_{k}$ to obtain compact surfaces with $\lambda_{1}$ bounded from below. The present method contrasts with the method of $[\mathrm{BBD}]$ in a number of ways. First of all, the surfaces $S_{C}$ obtained here can in general have large injectivity radius, as we show to be the case with the surfaces $P_{k}$, so the compact surfaces $S_{C}$ which can arise from this construction can reach parts of the moduli space of surfaces not accessible by the methods of [BBD]. This point of view is developed at length in the paper [TS].

Secondly, the method of [BBD] and the present paper can be used together to construct families of surfaces of varying large genus whose Cheeger constants, and hence first eigenvalues, are bounded uniformly from below, by applying the present method to some of the cusps and the method of [BBD] to the remaining cusps. We will pursue this line of thought in detail elsewhere.

\section{Some curvature calculations}

We begin by considering two metrics $d s_{D}^{2}$ and $d s_{C}^{2}$ on the punctured hyperbolic plane $\mathbb{H}^{2}-$ pt. The metric $d s_{D}^{2}$ is the standard hyperbolic metric on $\mathbb{H}^{2}$. If we write the punctured hyperbolic plane as the punctured unit disk

$$
D^{*}=\{z \in \mathbb{C}: 0<|z|<1\},
$$

then the metric $d s_{D}^{2}$ may be written as

$$
d s_{D}^{2}=\left[\frac{2}{1-r^{2}}\right]^{2}\left[d x^{2}+d y^{2}\right],
$$

where we have set $r=|z|$.

The metric $d s_{C}^{2}$ may be described as the unique metric in the standard conformal class which is complete on $\mathbb{H}^{2}$ - pt and has constant curvature -1 . It may be realized by taking the quotient $C$ of the standard hyperbolic metric on the upper half-plane $\mathbb{H}^{2}$ given by

$$
d s^{2}=\frac{1}{y^{2}}\left[d x^{2}+d y^{2}\right]
$$

by the isometry $A: z \rightarrow z+1$, and by identifying the quotient $\mathbb{H}^{2} / A$ with $D^{*}$ by the map

$$
z \rightarrow e^{2 \pi i z} .
$$

From this, it is easy to write out the explicit expression for $d s_{C}^{2}$ given by

$$
d s_{C}^{2}=\left[\frac{1}{r \log (r)}\right]^{2}\left[d x^{2}+d y^{2}\right] .
$$


The main goal of this section is the following:

Lemma 1.1. For every $\varepsilon$, there exists an $R$ and a metric $d s_{R}^{2}$ on $D^{*}$ with the following properties:

(i) $d s_{R}^{2}$ is conformally equivalent to $d s_{D}^{2}$ (and hence also $d s_{C}^{2}$ ) on $D^{*}$.

(ii) Outside a ball of radius $R$ about 0 in the metric $d s_{D}^{2}$, ds $s_{R}^{2}$ agrees with the metric $d s_{C}^{2}$.

(iii) The curvature of the metric $d s_{R}^{2}$ is everywhere between $-\left(\frac{1}{1+\varepsilon}\right)$ and $-(1+\varepsilon)$.

(iv) $d s_{R}^{2}$ extends across $z=0$ to give a smooth metric on $D=\{z:|z|<1\}$.

We begin the proof by considering radially symmetric metrics on $D^{*}$ of the form

$$
d s_{f}^{2}=f^{2}(r)\left[d x^{2}+d y^{2}\right]=f^{2}(r)\left[d r^{2}+r^{2} d \theta^{2}\right] .
$$

The curvature $K_{f}$ of the metric $d s_{f}^{2}$ is given by the formula

$$
K_{f}=\frac{-\left[\left(\frac{f^{\prime}}{f}\right)^{\prime}+\frac{f^{\prime}}{r f}\right]}{f^{2}} .
$$

Setting $K_{f}=-1$, we have the solutions

$$
f_{1}(r)=\frac{2}{1-r^{2}}
$$

corresponding to $d s_{D}^{2}$ and

$$
f_{2}(r)=\frac{-1}{r \log (r)}=\frac{1}{r \log (1 / r)}
$$

corresponding to $d s_{C}^{2}$.

We will need some simple facts about $f_{1}$ and $f_{2}$ :

Lemma 1.2. $f_{1}$ and $f_{2}$ satisfy the following:

(a) $\lim _{r \rightarrow 1} \frac{f_{2}}{f_{1}}=1$.

(b) $f_{2}>f_{1}$.

Proof. We first observe that as $r \rightarrow 1$, both $f_{1}$ and $f_{2}$ blow up. Hence, by L'Hospital's rule,

$$
\begin{aligned}
\lim _{r \rightarrow 1} \frac{f_{2}}{f_{1}} & =\lim _{r \rightarrow 1} \frac{\left(\frac{1}{f_{1}}\right)}{\left(\frac{1}{f_{2}}\right)} \\
& =\lim _{r \rightarrow 1} \frac{\left(1-r^{2}\right)^{\prime}}{-2(r \log (r))^{\prime}} \\
& =\lim _{r \rightarrow 1} \frac{-2 r}{-2(\log (r)+1)}=1 .
\end{aligned}
$$


This establishes (a).

(b) amounts to the assertion that

$$
1-r^{2}>-2 r \log (r)
$$

At 1 , both sides are equal to 0 , so this inequality will follow from the inequality

$$
-2 r<-2(\log (r)+1)
$$

or

$$
r>1+\log (r) .
$$

Again, we get equality at $r=1$, so the assertion will follow from

$$
1<1 / r
$$

which holds when $r<1$.

We now transform the problem of constructing the metrics $d s_{R}^{2}$ from a conformal problem on the unit disk to a problem of metrics of the form

$$
d s_{g}^{2}=g^{2}(r)\left[d r^{2}+\sinh ^{2}(r) d \theta^{2}\right] .
$$

The curvature of this metric is given by

$$
\kappa_{g}=\frac{-\left[\left(\frac{g^{\prime}}{g}\right)^{\prime}+1+\frac{g^{\prime}}{g} \operatorname{coth}(r)\right]}{g^{2}} .
$$

When $g \equiv 1$, we obtain the standard hyperbolic metric $d s_{D}^{2}$. It follows from our calculations above that the metric $d s_{C}^{2}$ is given by the function

$$
h(r)=\frac{f_{2}}{f_{1}}(R(r)),
$$

where $R(r)=\tanh (r / 2)$ is the Euclidean distance from 0 of a point whose hyperbolic distance from 0 is $r$. We thus have

$$
h(r)=\frac{1}{\sinh (r) \log (\operatorname{coth}(r / 2))} .
$$

It follows from Lemma 1.2 that $h(r) \rightarrow 1$ as $r \rightarrow \infty$, and that $h(r)>1$. We will need some more properties of $h$ :

Lemma 1.3. $h(r)$ has the following additional properties:

(a) $h^{\prime}(r)$ is negative and tends to 0 as $r \rightarrow \infty$.

(b) $h^{\prime \prime}(r)$ is positive and tends to 0 as $r \rightarrow \infty$. 
Proof. It is easily seen that $h^{\prime}$ is negative if and only if the same is true of its logarithmic derivative.

We may then compute

$$
\begin{aligned}
\log (h(r))^{\prime} & =-\left[\frac{\cosh (r)}{\sinh (r)}+\frac{(\operatorname{coth}(r / 2))^{\prime}}{\operatorname{coth}(r / 2) \log (\operatorname{coth}(r / 2)}\right] \\
& =-\left[\frac{\cosh (r)}{\sinh (r)}-\frac{1}{\sinh (r) \log (\operatorname{coth}(r / 2))}\right] \\
& =-\frac{1}{\sinh (r) \log (\operatorname{coth}(r / 2))}[\cosh (r) \log (\operatorname{coth}(r / 2))-1]
\end{aligned}
$$

From the fact that the curvature $\kappa_{h}$ is equal to -1 , or by a direct calculation, we see that

$$
(\log (h))^{\prime \prime}=\left(h^{2}-1\right)-(\log (h))^{\prime} \operatorname{coth}(r) .
$$

We now claim that assertions (a) and (b) both follow from the assertion that $\cosh (r) \log (\operatorname{coth}(r / 2))-1$ is positive, and tends to 0 as $r \rightarrow \infty$. This is evident in part (a), while for part (b) we use the equation

$$
(\log (h))^{\prime \prime}=\frac{h^{\prime \prime}}{h}-\frac{\left(h^{\prime}\right)^{2}}{h^{2}}
$$

to establish that if $(\log (h))^{\prime \prime}$ is positive and tends to 0 as $r \rightarrow \infty$, then the same is true of $h$.

The fact that $\cosh (r) \log (\operatorname{coth}(r / 2))-1$ is positive and tends to 0 as $r \rightarrow \infty$ follows readily from L'Hospital's Rule, as above.

This proves Lemma 1.3.

We will now prove Lemma 1.1 according to the following scheme: it is evident from the formula for curvature that, for any $\varepsilon$, there exists a $\delta$ with the following property: let $h_{\varepsilon}$ be any function which satisfies the following conditions:

$$
\begin{array}{r}
1 \leq h_{\varepsilon} \leq 1+\delta \\
\left|h_{\varepsilon}^{\prime} \operatorname{coth}(r)\right| \leq \delta \\
\text { and } \\
\left|h_{\varepsilon}^{\prime \prime}\right| \leq \delta,
\end{array}
$$

then the metric

$$
d s_{h_{\varepsilon}}^{2}=h_{\varepsilon}^{2}\left[d r^{2}+\sinh ^{2}(r) d \theta^{2}\right]
$$

will have curvature between $-\left(\frac{1}{1+\varepsilon}\right)$ and $-(1+\varepsilon)$. We must demand as well that $h_{\varepsilon} \rightarrow 1$ as $r \rightarrow 0$, in order to obtain a smooth metric at $r=0$. 
Given $\varepsilon$, we will then construct $h_{\varepsilon}$ as follows: let $k(r)$ be a smooth function which approximates the discontinuous function $k_{0}(r)$ defined by

$$
\begin{aligned}
k_{0}(r) & =0 & & \text { for } 0 \leq r<R_{0}-3 \\
& =c_{1} & & \text { for } R_{0}-3 \leq r<R_{0}-2 \\
& =-c_{1} & & \text { for } R_{0}-2 \leq r<R_{0}-1 \\
& =c_{2} & & \text { for } R_{0}-1 \leq r<R_{0} \\
& =h^{\prime \prime}(r) & & \text { for } r \geq R_{0},
\end{aligned}
$$

where we will choose $R_{0}, c_{1}$, and $c_{2}$ later.

In order to have the anitderivative $k_{1}(r)$ of $k_{0}$ with $k_{1}(0)=$ agree with $h^{\prime}(r)$ for $r \geq R_{0}$, we must have

$$
c_{2}=h^{\prime}\left(R_{0}\right) \text {. }
$$

We then let $k_{2}(r)$ be the antiderivative of $k_{1}(r)$ with $k_{2}(0)=1$. In order for this to equal $h(r)$ for $r \geq R_{0}$, we must have

$$
c_{1}=\left(h\left(R_{0}\right)-1\right)-\frac{c_{2}}{2}=\left(h\left(R_{0}\right)-1\right)-\frac{h^{\prime}\left(R_{0}\right)}{2} .
$$

One may then choose $k$ to be a smooth function approximating $k_{0}$, agreeing with $k_{0}$ for $R>R_{0}$, and satisfying the same conditions at $R_{0}$ as $k_{0}$. Our desired function $h_{\varepsilon}$ will then be the function which satisfies

$$
h_{\varepsilon}^{\prime \prime}=k, \quad h_{\varepsilon}^{\prime}(0)=0, \quad h_{\varepsilon}(0)=1 .
$$

We may then choose $R_{0}$ sufficiently large such that $\operatorname{coth}\left(R_{0}\right)$ and $h\left(R_{0}\right)$ are close to 1 , and $h^{\prime}\left(R_{0}\right), h^{\prime \prime}\left(R_{0}\right)$ are close to 0 .

This then completes the proof of Lemma 1.1.

\section{A comparison theorem}

Let $S_{O}$ be a Riemann surface with a complete metric $d s_{S_{O}}^{2}$ of finite area and constant curvature -1 . Then each cusp $C_{i}$ has a neighborhood which is isometric to a neighborhood of infinity in $C=\mathbb{H}^{2} /(z \sim z+1)$.

For $z$ in such a neighborhood, let $l(z)$ denote the length of the shortest closed horocycle through $z$. In terms of the coordinate $C$, we have that

$$
l(z)=\frac{1}{\Im(z)} .
$$

We may compactify $S_{O}$ to obtain a compact Riemann surface $S_{C}$ in the following way: for each cusp $C_{i}$, let

$$
B_{l}\left(C_{i}\right)=\left\{z \in C_{i}: l(z) \leq l\right\} .
$$


Then $B_{l}\left(C_{i}\right)$ is conformally equivalent to a punctured disk, with the equivalence given by the map $z \rightarrow e^{2 \pi i z}$.

We may then replace each neighborhood $B_{l}\left(C_{i}\right)$ with a solid disk to obtain $S_{C}$. This construction defines a unique conformal structure on $S_{C}$, and exhibits $S_{O}$ conformally as

$$
S_{O}=S_{C}-\left\{p_{1}, \ldots, p_{k}\right\} .
$$

Under the map $C \rightarrow D$ given by $z \rightarrow e^{2 \pi i z}$, the distance $r$ from $e^{2 \pi i z}$ to 0 in the hyperbolic metric on $D$ is related to $l(z)$ by

$$
l(z)=\frac{2 \pi}{\log \left(\frac{e^{r}+1}{e^{r}-1}\right)} .
$$

For each $p_{i} \in S_{C}$, let $d s_{S_{C}}^{2}$ denote the hyperbolic metric on $S_{C}$, assuming that $S_{C}$ carries such a metric, and let $B\left(r, p_{i}\right)$ denote the ball of radius $r$

Definition 2.1. The surface $S_{O}$ has cusps of length $\geq l$ if, for each $i$, there is a simple closed horocycle $h_{i}$ about the cusp $C_{i}$, such that each $h_{i}$ has length $\geq l$, and such that all the $h_{i}$ 's are disjoint,

In this section, we will prove:

Theorem 2.1. For every $\varepsilon$, there is an $l$ and $r$ such that, if $S_{O}$ has cusps of length $\geq l$, then outside of $\cup_{i} B_{l}\left(C_{i}\right)$ and $\cup_{i} B\left(r, p_{i}\right)$, we have

$$
\left(\frac{1}{1+\varepsilon}\right) d s_{S_{O}}^{2} \leq d s_{S_{C}}^{2} \leq(1+\varepsilon) d s_{S_{O}}^{2}
$$

Proof. Given $\varepsilon$, choose $R_{0}$ as in Lemma 1.1, and assume that the cusps of $S_{O}$ have length at least

$$
l_{0}=\frac{2 \pi}{\log \left(\frac{e^{R_{0}}+1}{e^{R_{0}}-1}\right)} .
$$

We may then replace the hyperbolic metric on each cusp by the conformally equivalent metric given by Lemma 1.1. The resulting metric then extends across the cusps to give a new metric $d s_{\varepsilon, R_{O}}^{2}$ on $S_{C}$ with the following properties:

(i) $d s_{\varepsilon, R_{O}}^{2}$ agrees with the hyperbolic metric on $S_{O}$ outside of $\cup_{i} B_{l_{0}}\left(C_{i}\right)$.

(ii) $d s_{\varepsilon, R_{O}}^{2}$ is conformally equivalent to the hyperbolic metrics on $S_{O}$ and $S_{C}$.

(iii) The curvatures of $d s_{\varepsilon, R_{O}}^{2}$ are everywhere between $-\left(\frac{1}{1+\varepsilon}\right)$ and $-(1+\varepsilon)$.

We now wish to compare the metric $d s_{\varepsilon, R_{O}}^{2}$ with the hyperbolic metric on $S_{C}$. This will be carried out using the following lemma of Wolpert [W], which is a generalization of the Ahlfors-Schwarz Lemma [A]: 
Lemma 2.1. ([W]) Let $S$ be a compact surfacce of genus at least 2. Let $d s^{2}$ and $d \sigma^{2}$ determine the same conformal structures. Provided the Gauss curvatures satisfy

$$
\kappa\left(d s^{2}\right) \leq \kappa\left(d \sigma^{2}\right)<0
$$

then $d s^{2} \leq d \sigma^{2}$.

To prove Theorem 2.1, we apply Lemma 2.1 to the metrics $(1+\varepsilon) d s_{\varepsilon, R_{O}}^{2}$ (resp. $\left.\left(\frac{1}{1+\varepsilon}\right) d s_{\varepsilon, R_{O}}^{2}\right)$ and $d s_{S_{C}}^{2}$. Since $(1+\varepsilon) d s_{\varepsilon, R_{O}}^{2}$ has curvature satisfying

$$
\kappa\left((1+\varepsilon) d s_{\varepsilon, R_{O}}^{2}\right) \geq-1=\kappa\left(d s_{S_{C}}^{2}\right)
$$

and similarly

$$
\kappa\left(\left(\frac{1}{1+\varepsilon}\right) d s_{\varepsilon, R_{O}}^{2}\right) \leq \kappa\left(d s_{S_{C}}^{2}\right),
$$

we conclude that

$$
\left(\frac{1}{1+\varepsilon}\right) d s_{\varepsilon, R_{O}}^{2} \leq d s_{S_{C}}^{2} \leq(1+\varepsilon) d s_{\varepsilon, R_{O}}^{2} .
$$

Since $d s_{\varepsilon, R_{O}}^{2}$ agrees with $d s_{S_{O}}^{2}$ outside the cusp neighborhoods $B_{l_{0}}\left(C_{i}\right)$, we have the same inequality with the metric $d s_{\varepsilon, R_{O}}^{2}$ replaced by $d s_{S_{O}}^{2}$ outside these neighborhoods. Furthermore, the image of the neighborhood $B_{l_{0}}\left(C_{i}\right)$ is contained in the ball $B\left(R_{1}, p_{i}\right)$ computed in the metric $d s_{\varepsilon, R_{O}}^{2}$, where $R_{1}=(1+\varepsilon) R_{0}$. But this ball is contained in the ball of radius $(1+\varepsilon)^{1 / 2} R_{1}$ computed in the metric $d s_{S_{C}}^{2}$, by the above inequality.

We may now take $r=(1+\varepsilon)^{1 / 2} R_{1}$ to complete the proof of Theorem 2.1.

We remark that this argument shows as well that the image of $B_{l_{0}}\left(C_{i}\right)$ contains $B\left(\frac{1}{(1+\varepsilon)^{3 / 2}} R_{0}, p_{i}\right)-p_{i}$.

\section{Counting short geodesics}

In this section, we will relate the lengths of short geodesics on $S_{C}$ with the lengths of short geodesics on $S_{O}$. We then use this to give a proof of Theorem 0.2.

We first observe that if $\gamma$ is a closed geodesic on $S_{O}$, then its image in $S_{C}$ is shorter, by the standard Schwarz Lemma, and hence the geodesic representing it will be still shorter. It may indeed be a great deal shorter, and even nullhomotopic.

We will, however, give a bound for lengths of geodesics on $S_{C}$ in terms of lengths of geodesics on $S_{O}$ of the following form:

Lemma 3.1. For $l$ sufficiently large, there is a constant $\delta(l)$ with the following property: Let $S_{O}$ have cusps of length $\geq l$. Then, for every geodesic $\gamma$ in $S_{C}$, there 
is a geodesic $\gamma^{\prime}$ in $S_{O}$, such that the image of $\gamma^{\prime}$ in $S_{C}$ is homotopic to $\gamma$, and

$$
\text { length }(\gamma) \leq \text { length }\left(\gamma^{\prime}\right) \leq(1+\delta(l)) \operatorname{length}(\gamma) .
$$

Furthermore, $\delta(l) \rightarrow 0$ as $l \rightarrow \infty$.

The idea of the proof may be paraphrased as follows: we will choose an $r_{2}$ larger than the $r$ of Theorem 2.1, such that any geodesic which enters $B\left(r_{2}, p_{i}\right)$ can be "pushed out of the way" to avoid $B\left(r, p_{i}\right)$. The increase in length of the curve will then be small compared to the legth involved in going from the boundary of $B\left(r_{2}, p_{i}\right)$ to the boundary of $B\left(r, p_{i}\right)$. The image of this "pushed away geodesic" in $S_{O}$ will then give the homotopy class for $\gamma^{\prime}$.

We will need the following elementary:

Lemma 3.2. Given $\delta_{1}$ and $r_{1}$, there is an $r_{2}$ with the following property: let $\gamma$ be any curve in the ball $B\left(r_{2}, x_{0}\right)$ of radius $r_{2}$ in the hyperbolic plane $\mathbb{H}^{2}$, whose endpoints lie in the boundary of $B\left(r_{2}, x_{0}\right)$ Then there is a curve $\tilde{\gamma}$ homotopic to $\gamma$ with a homotopy fixing the endpoints, such that $\tilde{\gamma}$ does not meet the ball $B\left(r_{1}, x_{0}\right)$, and

$$
\text { length }(\tilde{\gamma})<\left(1+\delta_{1}\right) \operatorname{length}(\gamma) \text {. }
$$

Proof. Indeed, we may choose $\tilde{\gamma}$ to agree with $\gamma$ up to the first time $\gamma$ enters $B\left(r_{1}, x_{0}\right)$ and after the last time $\gamma$ exits $B\left(r_{1}, x_{0}\right)$, and to travel around the perimeter of $B\left(r_{1}, x_{0}\right)$ from the entry point to the exit point. Choosing $r_{1}$ such that the length $l\left(r_{1}\right)$ of the perimeter of $B\left(r_{1}, x_{0}\right)$ satisfies

$$
\frac{l\left(r_{1}\right)}{2\left(r_{2}-r_{1}\right)}<\delta_{1}
$$

certainly gives $r_{2}$ with the desired properties.

We now can complete the proof of Lemma 3.1 as follows: Given $\delta$, let us write

$$
1+\delta=\left(\sqrt{1+\varepsilon_{1}}\right)\left(1+\delta_{1}\right)
$$

for some $\varepsilon$ and $\delta_{1}$. We then choose $r_{1}$ as in Lemma 2.1 and $r_{2}$ as in Lemma 3.1. Then, if the cusps of $S_{O}$ have length $\geq l$, where $l$ is sufficiently large so that the images of the $B_{l}\left(C_{i}\right)$ 's all lie within the corresponding $B\left(r_{2}, p_{i}\right)$ 's, then we may modify the curve $\gamma$ to a curve $\tilde{\gamma}$ which does not meet any $B\left(r_{1}, p_{i}\right)$, increasing its length by a factor of at most $1+\delta_{1}$. When we now measure the curve $\tilde{\gamma}$ in the metric $d s_{S_{O}}^{2}$, its length increases by a factor of at most $\sqrt{1+\varepsilon_{1}}$. If we denote by $\gamma^{\prime}$ the geodesic in the homotopy class of $\tilde{\gamma}$ in $S_{O}$, then we clearly have that

$$
\operatorname{length}\left(\gamma^{\prime}\right) \leq(1+\delta) \operatorname{length}(\gamma)
$$

The inequality length $(\gamma) \leq \operatorname{length}\left(\gamma^{\prime}\right)$ then follows from the Ahlfors-Schwarz Lemma, as mentioned above. 
This concludes the proof of Lemma 3.1.

We will now prove Theorem 0.2. As indicated in the introduction, it will follow from the Theorem of Huxley [Hu] and Sarnak-Xue [SX], see also [TFSG] for a discussion.

Suppose that $R_{k}$ is a family of Riemann surfaces, such that $P S L(2, \mathbb{Z} / k)$ acts on $R_{k}$. We then have:

Theorem 3.1. ([Hu], $[\mathrm{Sx}])$ Suppose that there are constants $c_{1}, c_{2}$, and $c_{3}$, and for all $\varepsilon>0$ a constant $c_{4}(\varepsilon)$ such that:

(a) $c_{1} k^{3} \leq \operatorname{vol}\left(R_{k}\right) \leq c_{3} k^{3}$.

(b) If $f_{k}$ is an eigenfunction of the Laplacian on $R_{k}$ invariant under the action of $\operatorname{PSL}(2, \mathbb{Z} / k)$ with eigenvalue $\lambda$, then $\lambda>5 / 36$.

(c) For all $\varepsilon$, the number of geodesics of length $\leq(6-\varepsilon) \log (k)$ on $R_{k}$ is at most $c_{4}(\varepsilon) k^{6+\varepsilon}$.

Then

$$
\liminf _{k \rightarrow \infty} \lambda_{1}\left(R_{k}\right) \geq 5 / 36
$$

It is argued in detail in $[\mathrm{Hu}]$ that the surfaces $S_{k}=\mathbb{H}^{2} / \Gamma(k)$ satisfy these conditions. The only non-trivial part is to verify (c). This is done with an explicit calculation with traces of matrices satisfying the congruence condition.

We now turn our attention to showing that (a)-(c) obtain for the surfaces $P_{k}$ as well.

Observing that the quotient of $P_{k}$ by $P S L(2, \mathbb{Z} / k)$ is the hyperbolic triangle $T_{k}$ with angles $\pi / 3, \pi / 3$, and $2 \pi / k$, while the quotient of $S_{k}$ by $P S L(2, \mathbb{Z} / k)$ is the hyperbolic triangle with angles $\pi / 3, \pi / 3$, and one ideal vertex, we see that

$$
\operatorname{vol}\left(P_{k}\right)=(1-6 / k) \operatorname{vol}\left(S_{k}\right)
$$

from which (a) follows immediately.

Furthermore, if $f_{k}$ is an eigenfunction with eigenvalue $\lambda$ on $P_{k}$ invariant under $P S L(2, \mathbb{Z} / k)$, then $f_{k}$ descends to a function on $T_{k}$ whose Rayleigh quotient is $\lambda$. The lower bound $\lambda \geq 1 / 4$ will then follow from Cheeger's inequality and the fact that the Cheeger constant $h^{N}\left(T_{k}\right)$ with Neumann boundary conditions is $\geq 1$.

But the fact that $h^{N}(T) \geq 1$ for any hyperbolic triangle is quite standard, see [Bu2], establishing (b).

To establish (c), we observe that the surfaces $S_{k}$ have cusps of length $\geq k$. Lemma 3.1 then allows us to deduce (c) for the surfaces $P_{k}$ from the analogous statement for the surfaces $S_{k}$.

This completes the proof of Theorem 0.2. 


\section{The Cheeger constant}

We first recall the Cheeger constant $h(S)$ of a surface. It is given by

$$
h(S)=\inf _{C} \frac{\operatorname{length}(C)}{\min (\operatorname{vol}(A), \operatorname{vol}(B))},
$$

where $C$ runs over all curves dividing $S$ into two pieces $A$ and $B$.

According to the inequalities of Cheeger [Ch] and Buser $[\mathrm{Bu}]$, we have that

$$
(1 / 4) h^{2} \leq \lambda_{1}(S) \leq c_{1} h+c_{2} h^{2}
$$

where $c_{1}$ and $c_{2}$ depend on a lower bound for the curvature of $S$. In particular, it follows that, in the presence of a lower bound for the curvature, a bound for below for $\lambda_{1}$ is equivalent to a lower bound for $h$.

Of course, as is discussed in [SGCC], the loss of strength in passing from an estimate for the Cheeger constant to an estimate for $\lambda_{1}$ is significant, so that one does not expect the constants that one obtains in Theorem 0.2 from this approach. Indeed, it is shown in [SGCC] that the Cheeger constant $h\left(S_{k}\right)$ is too small to give Selberg's 3/16 bound for $\lambda_{1}\left(S_{k}\right)$. On the other hand, passing through the Cheeger constant allows us to obtain spectral estimates in more general situations than are allowed for by the approach of $\S 3$.

We will show:

Theorem 4.1. For $l$ sufficiently large, there is a constant $C(l)$ with the following property: if $S_{O}$ is a Riemann surface with cusps of length $\geq l$, then the Cheeger constants $h\left(S_{O}\right)$ and $h\left(S_{C}\right)$ satisfy

$$
\left(\frac{1}{C(l)}\right) h\left(S_{O}\right) \leq h\left(S_{C}\right) \leq C(l) h\left(S_{O}\right) .
$$

Furthermore, $C(l) \rightarrow 0$ as $l \rightarrow \infty$.

Proof. Let $\gamma$ be a curve in $S_{C}$ dividing $S_{C}$ into two pieces $A$ and $B$, such that the ratio

$$
\frac{\operatorname{length}(\gamma)}{\min (\operatorname{vol}(A), \operatorname{vol}(B))}
$$

realizes the Cheeger constant. We may assume that $\operatorname{vol}(A) \leq \operatorname{vol}(B)$.

As in Lemma 3.1, if $l$ is sufficiently large, we may choose $r_{1}$ and $r_{2}$ such that $\gamma$ may be pushed away from the neighborhoods $B\left(r_{1}, p_{i}\right)$ to obtain a new curve $\tilde{\gamma}$ whose length is at most $(1+\delta(l)$ length $(\gamma)$.

In fact, we have a choice of how to push $\gamma$. For each $i$, we may consider the neighborhoods $B\left(r_{2}, p_{i}\right)$ and the sets

$$
A_{i}=A \cap B\left(r_{2}, p_{i}\right), \quad B_{i}=B \cap B\left(r_{2}, p_{i}\right)
$$


If $\gamma$ does not meet $B\left(r_{1}, p_{i}\right)$, then we do not change $\gamma$ in $B\left(r_{2}, p_{i}\right)$. Otherwise, we may push $\gamma$ so that, for each $i$, if $\operatorname{vol}\left(A_{i}\right) \leq \operatorname{vol}\left(B_{i}\right)$, then $\tilde{\gamma}$ divides $B\left(r_{2}, p_{i}\right)$ into two pieces $A_{i}^{\prime}, B_{i}^{\prime}$ with

$$
A_{i}^{\prime}=A_{i} \cup B\left(r_{1}, p_{i}\right), \quad B_{i}^{\prime}=B_{i}-B\left(r_{1}, p_{i}\right) .
$$

Similarly, if $\operatorname{vol}\left(B_{i}\right) \leq \operatorname{vol}\left(A_{i}\right)$, then we choose $\tilde{\gamma}$ so that

$$
B_{i}^{\prime}=B_{i} \cup B\left(r_{1}, p_{i}\right), \quad A_{i}^{\prime}=A_{i}-B\left(r_{1}, p_{i}\right) .
$$

We now claim that $\tilde{\gamma}$ divides $S_{C}$ into two pieces $A^{\prime}$ and $B^{\prime}$ with

$$
\operatorname{vol}\left(A^{\prime}\right) \geq\left(1-\varepsilon^{\prime}\right) \operatorname{vol}(A), \quad \operatorname{vol}\left(B^{\prime}\right) \geq\left(1-\varepsilon^{\prime}\right) \operatorname{vol}(B),
$$

with

$$
\varepsilon^{\prime}=2 \frac{\operatorname{vol}\left(B\left(r_{1}, p_{i}\right)\right)}{\operatorname{vol}\left(B\left(r_{2}, p_{i}\right)\right)}
$$

This is clear, since the only times a piece is taken from $A_{i}$ (resp. $B_{i}$ ) is when $\operatorname{vol}\left(A_{i}\right)$ is larger than $(1 / 2) \operatorname{vol}\left(B\left(r_{2}, p_{i}\right)\right.$.

We now regard $\tilde{\gamma}$ as a curve in $S_{O}$, and compute

$$
\frac{\operatorname{length}(\tilde{\gamma})}{\min \left(\operatorname{vol}\left(A^{\prime}\right), \operatorname{vol}\left(B^{\prime}\right)\right)}
$$

in the metric $d s_{S_{O}}^{2}$.

But in passing from the metric $d s_{S_{C}}^{2}$ to the metric $d s_{S_{O}}^{2}$, the length of $\tilde{\gamma}$ is multiplied by a factor of at most $\sqrt{1+\varepsilon}$, while the volumes of the parts of $A^{\prime}$ and $B^{\prime}$ not meeting $B\left(r_{1}, p_{i}\right)$ are divided by at most $1+\varepsilon$. Also, the balls $B\left(r_{1}, p_{i}\right)$ have larger volume in the metric $d s_{S_{O}}^{2}$ than in the metric $d s_{S_{C}}^{2}$, as follows from the Schwarz inequality, or can be seen directly.

We thus have that

$$
\frac{\operatorname{length}(\tilde{\gamma})}{\min \left(\operatorname{vol}\left(A^{\prime}\right), \operatorname{vol}\left(B^{\prime}\right)\right)} \leq \frac{(1+\varepsilon)^{3 / 2}(1+\delta)}{1-\varepsilon^{\prime}} h\left(S_{C}\right) .
$$

$h\left(S_{O}\right)$ is less than the left-hand side, so we thus have

$$
h\left(S_{O}\right) \leq\left(C_{1}(l)\right) h\left(S_{C}\right),
$$

with

$$
C_{1}(l)=\frac{(1+\varepsilon)^{3 / 2}(1+\delta)}{\left(1-\varepsilon^{\prime}\right)} .
$$

To obtain an inequality in the opposite direction, we proceed in the identical manner, switching the roles of $S_{O}$ and $S_{C}$. We must make the following changes in 
the argument: first of all, we must reprove Lemma 3.2 in the case of a punctured disk rather than a disk. The proof is identical, except we no longer demand that the resulting curve $\tilde{\gamma}$ is homotopic to $\gamma$. This allows us to retain the option of pushing $\gamma$ in either direction around the puncture.

Secondly, we need an estimate of the form

$$
\operatorname{vol}\left(B_{l_{1}}\right)_{d s_{S_{C}}^{2}} \geq\left(\operatorname{const}\left(l_{1}\right)\right)\left(\operatorname{vol}\left(B_{l_{1}}\right)_{d s_{S_{O}}^{2}}\right)
$$

But the volume of $B_{l_{1}}$ in the metric $d s_{S_{O}}^{2}$ is precisely $l_{1}$, while the metric of a ball of radius $r_{1}$ in the hyperbolic plane is $2 \pi\left(\cosh \left(r_{1}\right)-1\right)$. Choosing $r_{1}$ so that

$$
l_{1}=\frac{2 \pi}{\log \left(\frac{e^{r} 1+1}{e^{r} 1^{-1}}\right)}
$$

and using L'Hospital's rule, we see that

$$
\frac{\operatorname{vol}\left(B_{l_{1}}\right)}{\operatorname{vol}\left(B\left(r_{1}, p_{i}\right)\right)} \rightarrow 1 \text { as } l_{1} \rightarrow \infty
$$

Passing from the metric $d s_{S_{O}}^{2}$ to the metric $d s_{\varepsilon, R_{O}}^{2}$ and then to the metric $d s_{S_{C}}^{2}$ introduces some factors of $1+\varepsilon$ into this calculation to give us the desired estimate.

Putting these together, we find a constant $C_{2}(l)$ such that

$$
h\left(S_{C}\right) \leq C_{2}(l) h\left(S_{O}\right)
$$

with $C_{2}(l) \rightarrow 1$ as $l \rightarrow \infty$.

This then concludes the proof of Theorem 4.1.

\section{Acknowledgments}

The work on this paper was begun while the author was a Fulbright scholar visiting Hebrew University in the 1993-94 academic year, and was completed under a Guastella fellowship at the Technion. We would like to thank these departments for their hospitality.

We greatly benefitted from conversations with a number of people, including Ron Donagi, Sasha Reznikov, and Paul Schmutz. We would like to extend our gratitude to them.

We also thank the referee for his comments on an earlier version of this paper, and for the opportunity to rewrite it. 


\section{References}

[A] L. Ahlfors, An extension of Schwarz's lemma, Trans. AMS 43, 359-364.

[SGCC] R. Brooks, Spectral geometry and the Cheeger constant. In: J. Friedman (ed.), Expanding Graphs, Proc. DIMACS Workshop, AMS (1993), pp. 5-19.

[TFSG] R. Brooks, Trace-formula methods in spectral geometry, J. Fourier Anal. App. (1995), 87-95.

[TS] R. Brooks, Twist surfaces, to appear Proc. Cortona Conf.

[BFK] R. Brooks, H. Farkas, and I. Kra, Number theory, theta identities, and modular curves. In: Quine and Sarnak (eds), Extremal Surfaces, Contemp. Math. 201 (1997), 125-154.

[BBD] P. Buser, M. Burger, and J. Dodziuk, Riemann surfaces of large genus and large $\lambda_{1}$. In: T. Sunada (ed), Geometry and analysis on Manifolds, Lecture Notes in Math 1339 (1988), pp. 54-63.

[Bu] P. Buser, A note on the isoperimetric constant, Ann. Sci. Ec. Norm. Sup. 15 (1982), 213-230.

[Bu2] P. Buser, On Cheeger's inequality $\lambda_{1} \geq h^{2} / 4$. In: Osserman and Weinstein (eds), Geometry of the Laplace Operator, Proc. Symp. Pure Math XXXVI (1980), pp. 29-77.

[Ch] J. Cheeger, A lower bound for the smallest eigenvalue. In: Gunning (ed), Problems in Analysis, Princeton University Press 1970, pp. 195-199.

$[\mathrm{Hu}]$ M. N. Huxley, Exceptional eigenvalues and congruennce subgroups. In: Hejhal, Sarnak, and Terras (eds), The Selberg Trace Formula and Related Topics, Contemp. Math 53 (1986), 341-349.

[SX] P. Sarnak and X. Xue, Bounds for multiplicities of automorphic representations, Duke Math J. 64 (1991), 207-227.

[Se] A. Selberg, On the estimation of Fourier coefficients of modular forms. In: A. L. Whiteman (ed.), Theory of Numbers, Proc. Symp. Pure Math 8 (1965), 1-15.

[W] S. Wolpert, A generalization of the Ahlfors-Schwarz lemma, Proc. AMS 84 (1982), 377-378.

Robert Brooks

Department of Mathematics

The Technion - Israel Institute of Technology

Haifa, Israel

e-mail: rbrooks@tx.technion.ac.il

(Received: November, 1996; revised: February, 1998) 